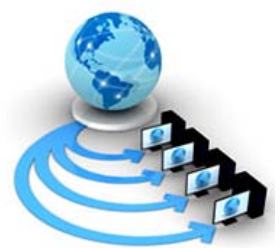

International Journal of Advanced Research in Computer Science

RESEARCH PAPER

\author{
Available Online at www.ijarcs.info
}

\title{
HYBRID MULTIDISCIPLINARY ALGORITHM FOR REAL TIME PROJECTILE MOTION TRACKING, LOGGING \& PREDICTIO
}

\author{
Abha Agrawal \\ Student (M.Tech- CS ) \\ Poornima College of Engineering, \\ Jaipur,India
}

\author{
Ajay Khuteta \\ Head of Department Computer Science \\ Poornima College of Engineering, \\ Jaipur, India
}

\begin{abstract}
Video reconnaissance is generally used to screen the place which needs steady security, for example, Banks, Shopping Malls, and Highways, military region, swarmed open spots, nation fringes and so forth. The significant debate incorporate the perplexing movement practices of various human items, complex scenes with various targets, recognition of progress in human movement. The goal of this paper is to build up a visual recognition and following arrangement of watching moving articles with the assistance of Hybrid Multidisciplinary Algorithm. The undertaking of movement recognition framework is to distinguish a "zone of movement" exhibit in a "region of condition being observed". Movement recognition is typically programming based checking calculation. In this part we lead the basic picture handling operations and caught them with the assistance of Hybrid Multidisciplinary Algorithm for Real Time Projectile Motion.

We propose the Method of following calculation which coordinates the versatile best foundation recognition, information affiliation, including new estimation, and straight task issue to limit the cost of perception of following. The exploratory outcome demonstrates that the dynamic foundation can be removed precisely and speedily, the calculation can be used in the continuous following applications.
\end{abstract}

Keywords: Real Time Projectile Motion, Active background estimation, Activity modelling, Data association, video monitoring, Linear Assignment problem, Hybrid Multidisciplinary Algorithm.

\section{INTRODUCTIONA}

Other name of hybrid algorithm is half and half algorithm. A half and half calculation is a calculation that joins at least two different calculations that take care of a similar issue, either picking one (contingent upon the information), or exchanging between them through the span of the calculation. This is by and large done to consolidate craved elements of every, so the general calculation is superior to the individual parts.

"Half and half calculation" does not allude to just joining various calculations to take care of an alternate issue numerous calculations can be considered as blends of easier pieces - however just to consolidating calculations that tackle a similar issue, yet vary in different attributes, remarkably execution. [2][3]

\section{Example:}

In software engineering, cross breed calculations are extremely normal in advanced genuine usage of recursive calculations, especially executions of separation and overcome or diminish and vanquish calculations, where the measure of the information diminishes as one moves further in the recursion. For this situation, one calculation is utilized for the general approach (on huge information), however somewhere down in the recursion, it changes to an alternate calculation, which is more proficient on little information. A typical illustration is in arranging calculations, where the inclusion sort, which is wasteful on extensive information, yet extremely effective on little information (say, five to ten components), is utilized as the last stride, after basically applying another calculation, for example, consolidate sort or quick sort. Consolidation sort and quick sort are asymptotically ideal on huge information, yet the overhead winds up noticeably noteworthy if applying them to little information, consequently the utilization of an alternate calculation toward the finish of the recursion. An exceptionally streamlined half breed arranging calculation is Time sort, which consolidates combine sort, inclusion sort, together with extra rationale (counting paired inquiry) in the blending rationale. [4][5]

\section{LITERATURE REVIEW}

Abhijit Paul Prabhat Ranjan Sahoo described that "The task of motion detection system is to detect an "area of motion" present in an "area of environment being monitored". Motion detection is usually software based monitoring algorithm. In this part we conduct the essential image processing operations. MATLAB'S Graphical User Interface Development Environment (GUIDE) provides a rich set of tools for lying out Graphical User Interfaces (GUI) in M-functions.

Motion detection is used to determine the trajectory of the projectile, its orientation relative to the plane, its velocity and its spin. It is very useful in detecting the presence of any projectile in case of high speed video.

Motion detection:

Motion detection is a process of confirming a change in position of an object relative to its surroundings or the change in the surroundings relative to an object. The task of a motion detection system is to detect a "area of motion" present in a "area of environment being monitored". The area of motion in this case refers to portion of the environment with activity due to the motions of the moving objects. Motion detection is usually software based monitoring algorithm which, when it detects motions will signal the surveillance camera to begin 
capturing the event or simply shows the motion detection using graphical method.

successfully detected the motion from a given image sequence extracted from a video .The method we have implemented work good in case of a sill background but in case of changing background it shows some deviation. [1]

\section{PROPOSED WORK}

1. Design \& Development of a hybrid multidisciplinary algorithm/software to track and or predict motion of a projectile.

2. Use of HSV color space in conjunction with DWT (Discrete Wavelet Transform) technique achieves higher background immunity \& contrast independence.

3. Use of artificial intelligence tools such as Artificial Neural Network to predict (not calculate) projectile trajectory in real time using previous learning experiences.

4. Possibility of real time projectile motion tracking system using low cost webcam \& MATLAB image processing toolbox.

5. Extraction of features of projectile motion such as velocity, distance, time etc, in real time using frame size as spatial reference \& frame rate as time reference.

6. Data logging to disk with timestamp \& image stamp for projectile parameters, for further analysis \& review.

7. Possibility of tracking multiple projectiles in motion using modified motion detection algorithm, to spatially separate motion detection regions.

\section{METHODOLOGY}

There are two fundamental sorts of strategies for movement location

\section{A. Edge Differencing Method}

\section{B. Foundation Subtraction Method}

\section{A. Edge Differencing Method}

The outright contrast between progressive edges can be utilized to separate picture popularity into changed and unaltered locales. Since just the protest moves, we anticipate that the changed district will be connected just with the question or conceivably its shadow.

\section{B. Foundation Subtraction Method}

Another way to deal with following the question foundation picture and subtract it from approach here is to discover the pixel-wise a few neighboring casings. That is morphological expansion does, on the off chance that you utilize a storiented along the edge measurement.

In the event that we watch some protest from far separation, we consider its gross elements rather than fine points of interest. On the off chance that that question is having distinctive hues on its diverse parts then the shading which is involving greatest zone of that protest is considered, and it is said that question that protest is of that shading. e.g. indicates protest is having green shading covering the majority of its parts, and blue and dark hues covering just some of its bit. So shade of that question is thought to be green as it were. To discover shade of the protest, shading picture got from camera is legitimately ANDed with pre-handled picture. This subsequent picture is changed over into HSV picture. Shade plane of HSV picture contains just shading data. Every one of the estimations of Hue design lies between 0 to 1 . Contingent on their qualities, shading is recognized. E.g. Red, green and blue hues can be recognized as in HSV plane as red pixels have values $>0.9$ and $<0.15$, green pixels have values $>0.15$ however $<0.49$, blue pixels have values $>0.49$ yet $<0.9$. In this manner second gross [6]

Highlight of the articles that is its shading is distinguished. Shading and shape discovery is likewise performed on database pictures. In the event that match between any on the database protest and meddling item is discovered then that question is said to be perceived. What's more, it must be demolished. [7]

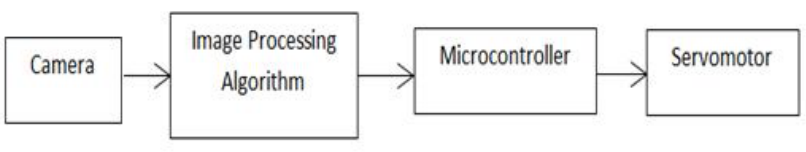

Fig 1.1 Block diagram

RESULT

\section{A. RGB Picture:}

To finish up in the event that we are including hues we picture while not including shading produces essential hues speak to absence of some of shading models.

\section{B. Dim Picture:}

These handling me power of single pixels. Helpful in various application pictures and shooting a film with utilizing this idea.

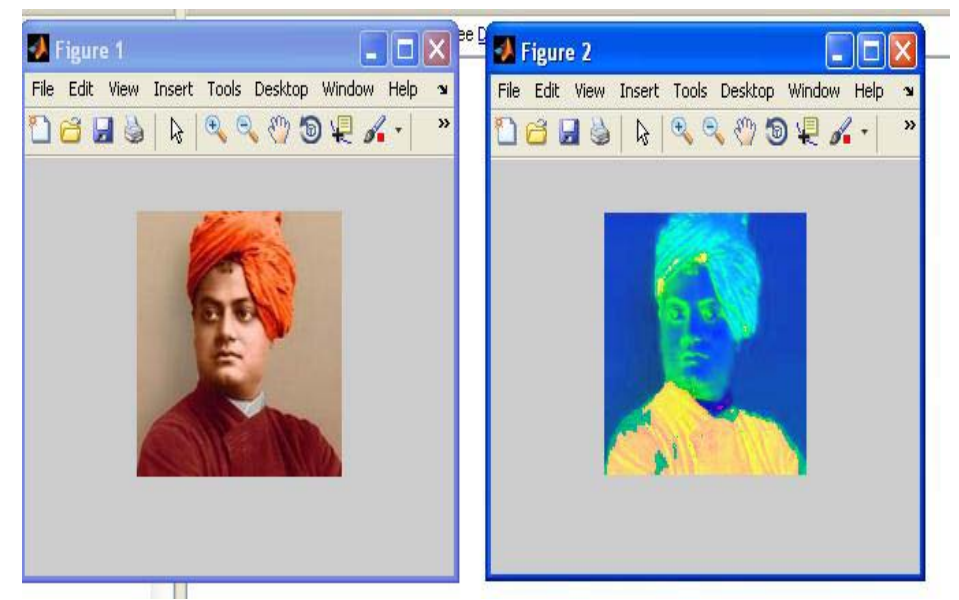

Fig. 1.2 read RGB object

C. Read the Video:

Read the video document we space show. 

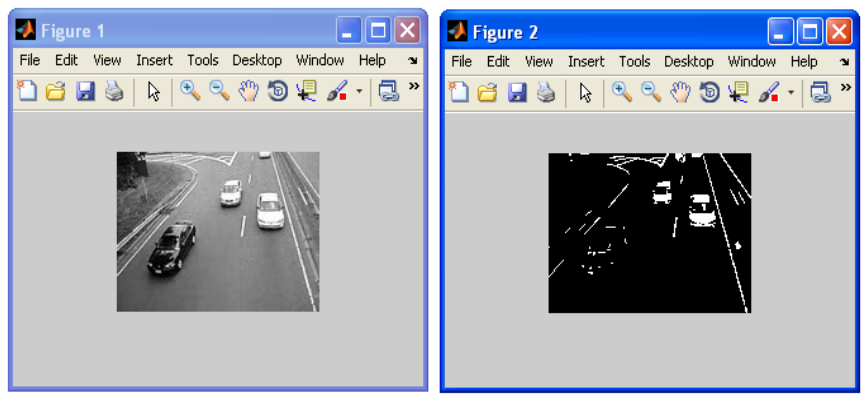

Fig.1.3 Read the Object in a Video

D. Discovery of Object:

Discovery of the protest in a dark scale show.

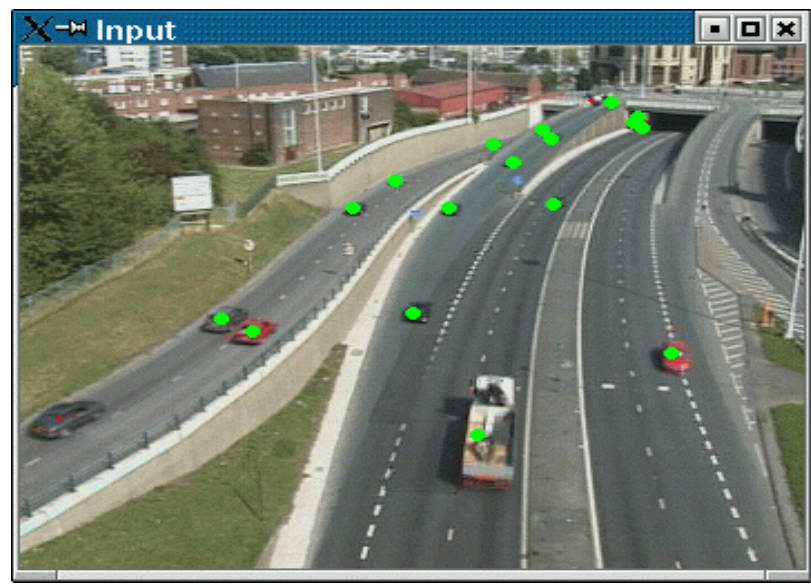

Fig.1.4 Recognize the Object in a dim scale

Techniques are construct just in light of the negatives of computerized pictures are, for example, showing medicinal green with monochrome positive coming about negatives as ordinary.

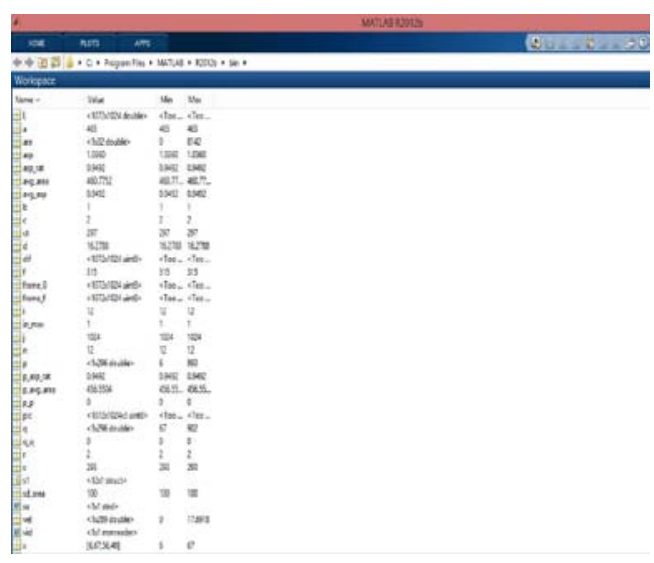

Fig. 1.5All the data running

\section{CONCLUSION}

Along these lines, at last we effectively distinguished the movement from a given picture grouping extricated from a video .The strategy we have actualized work great in the event of a ledge foundation yet in the event of changing foundation it demonstrates a few deviation. A video checking and recognition framework was in this way created effectively in this paper. This framework for the most part gives an productive technique for observation purposes and is planned to be exceptionally valuable for any individual or association. In this way, a movement based change location in avi video design was finished and effectively actualized. The future extent of the work done could be as per the following: the proper method of time as we began to comprehend the moment points of interest of our work, we essentially understood that our product would be hugely critical later on world. [9][10]

\section{ACKNOWLEDGMENT}

We thank to Prof. xxxxx, Department of Electronics and Communication Engineering, $\operatorname{xxxxxxx}$ for extending the support during Course of this investigation.

We are highly grateful to all those, who directly and indirectly helped us and who evinced keen interest and invaluable support in the progress and successful completion of our project work.

\section{REFERENCES}

[1] GUI Based Motion Detection of a Projectile Abhijit Paul Prabhat Ranjan School Dept. of ECE, Centurion University of Technology \& Management, Odisha, India

[2] S. Sulaiman, A. Hussain, N. Md. Tahir and S.A. Samad. "Graphical User Interface (GUI) Development for Object Tracking System in Video Sequences”. World Applied Sciences Journal 4 (2): 244-249, 2009.

[3] Debabrata Debnath, Arijit Choudhury, Abhisek Chakraborty, Ashok ku. Ray, Swanibhar Majumder."MATLAB Based Motion Detection Circuit”. Proceeding of Third National Conference on Mathematical Techniques: Emerging Paradigms For Electronics \& IT Industries (MATEIT)”, 2011.

[4] Raman Maini and Himanshu Aggarwal. "A Comprehensive Review of Image enhancement techniques”. journal of computing, volume 2, issue 3, march 2010, issn 2151-9617

[5] A.Harish Kumar Gupta, B.Pooja Upadhyay, c.sourabh dubey, f.d.satyendra pratap sing. "motion security/theft protection using mat lab programming”. International journal of advance technology \& engineering research (ijater), 2013.

[6] He, D.; Hujic, D.; Mills, J.K.; Benhabib, B. "Moving-object recognition using premarking and active vision "; Robotics and Automation, 1996. Proceedings. 1996 IEEE International Conference.

[7] Diplaros, A.; Gevers, T.; Patras, I.; "Combining color and shape information for illumination-viewpoint invariant object recognition",Image Processing, IEEE Transactions on Jan. 2006 Volume : 15 ,Issue: 1

[8] Jae-Han Park; Seung-Ho Baeg; Jaehan Koh; Kyung-Wook Park;Moon-Hong Baeg; "A new object recognition system for service robots in the smart environment" Control, Automation and Systems,2007. ICCAS '07.

[9] Chyi-Yeu Lin; Setiawan, E. "Object orientation recognition based on SIFT and SVM by using stereo camera", Robotics and Biomimetics, 2008. ROBIO 2008. IEEE International Conference.

[10] Chensheng Wang; Fei Wang; "A Knowledge-Based Strategy for Object Recognition and Reconstruction "; Information technology and Computer Science, 2009. itcs 2009. 